\title{
Zur Herkunft einiger ugrischer Wörter
}

Matti Liimola hat in seiner Untersuchung "Wogulische Ableitungssuffixe» (FUF XXX 248 ff.) in seiner gründlichen Art u.a. das wogul. Nominalsuffix $-t u l$ behandelt. Er kommt m.E. zu einem gut begründeten interessanten Ergebnis: es handle sich um »ein schönes Beispiel für die Entwicklung eines Substantivs zum Ableitungssuffix». Als Ausgangspunkt des offenbar ganz jungen Suffixes wird (S. 267) folgende wogul. Gefässbenennung angegeben: $L U t \bar{u} l$ 'viereckige trogartige Speiseschüssel aus Holz, wird auf der Jagd und beim Fischfang ange-

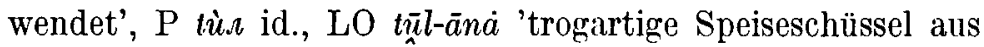
Holz' (āna 'Napf, Schale'), (?) KU tulppö̈̇ $\ddot{a}_{o} r t$ 'in seiner Mitte in der Längsrichtung gebogenes Brettstück, das man quer in das Boot legt und worauf man die Netze hebt' (pö̈̇̈̈rt 'Brett'), (?) TJ tŏspì̀ 'aus der Kante eines alten Bootes hergestelltes Fischbrett, worauf man beim Fischfang Fische aus dem Topfe schöpft'. Steinitz meint, das Wort gehöre zı jenen Fällen, wo für das (Spät)urwogulische ein $* \bar{u}$ anzunehmen ist (Wog. Vok. 209).

Liimola erörtert die Herkunft des Substantivs tūl nicht; er begnügt sich mit dem Hinweis auf Munkácsis Meinung (ÁKE 586/7), wonach das wogul. Wort eine Entsprechung im Ungarischen besitzt: tál (seit dem 14. Jh. verzeichnet) 'Schüssel; Gericht'. Dies Wort war bereits durch Flórián Mátyás (1856) - einem fanatischen Verfechter des "Ariertums" der ungarischen Sprache - mit dem neupersischen Substantiv $t \bar{a} l$ verbunden worden, das im grossen "Lexicon persico-latinum etymologicum" (1855/64) von I. A. Vullers folgendermassen erklärt wird: 'discus s. paropsis ex ære, auro, argento, simil.; duo cymbala parva ærea, quæ musici Indici canentes et saltantes inter se concutere solent'. Auch Munkácsi hält (a.a.O.) die ungar. und wogul. Wörter ohne weiteres für iranischer Herkunft. 
Später hat u.a. Pais ohne Bedenken das wogul. Substantiv mit der ungar. Gefässbezeichnung zusammengestellt (MNy. 50 329); die ugrische Ausgangsform hätte *tāls lauten können. Bárczi führt ebenfalls ungar. tál zurück auf die ugrische Zeit und erwähnt von einer möglichen iranischen Herkunft nichts (SzófSz. 298). Erst Éva Korenchy hat die Frage der Entlehnung in ihrer Arbeit "Iráni eredetű szavak az obi-ugor nyelvekben" (1968; Manuskr., S. 108-110) erneut vorgenommen. Sie führt Munkácsis Meinung an und bemerkt dann, für das genannte neupersische Wort seien keine Entsprechungen aus anderen iran. Sprachen und auch keine sicheren Verwandten von indischer Seite angeführt worden. Die Verfasserin wundert sich ferner über die grosse Differenz der Bedeutungen: im Persischen ein Spezialterminus, der ein metallenes Schlaginstrument bezeichnet, ugrischerseits die Benennung eines Speisegefässes, ja sogar eines Brettstückes. Nach Éva Korenchys Meinung kann man die ungar. und wogul. Wörter nur mit Mühe für iranischer Herkunft halten; als Ausgangspunkt wäre dann ein valt- oder mitteliranisches *täl-» zu rekonstruieren.

Jenes neupersische seltene $t \bar{a} l \mathrm{im}$ Wörterbuch von Vullers ist denn doch nicht so einzigartig wie die Fennougristen vermutet haben. Das Wort ist in zahlreichen iranischen, indischen und dardischen Sprachen bekannt und bezeichnet nicht nur ein metallenes Schlaginstrument sondern auch ein Gefäss.

Iranisch: pašto tāl 'медный таз' (M. G. Aslanov, Афганскорусск. словарь 212); sangleči tāl 'cooking-pot', yidghā $t a \bar{l}$, munji tōlo, tâla 'kettle' (G. Morgenstierne, Indo-Iranian Frontier Languages II 254, 415);

Dardisch: kati țōl 'dish, metal vessel' (Morgenstierne a.a.0.);

Indisch: sanskrit tāla-m. 'cymbal', pāli tāla-m. 'gong, cymbal', bengali $t \bar{a} l$ 'cymbal', hindi $t \bar{a} l \mathrm{f}$. 'small bell' (R. L. Turner, A Comparative Dictionary of the Indo-Aryan Languages, Nr. 5801; Mayrhofer notiert das Wort nicht in seinem altindischen etymologischen Wörterbuch).

Es ist wahrscheinlich, dass diese Bezeichnung für metallene Schlaginstrumente, "Becken», die offenbar indischen Ursprungs ist, relativ spät in die dardischen und iranischen Sprachen gelangte und dann auch die Bedeutungen eines metallenen Ge- 
fässes und allgemein eines Gefässes, einer Schüssel erhielt, obwohl der ursprüngliche indische Gehalt des Wortes auch im Neupersischen noch deutlich sichtbar ist. In die ugrischen Sprachen gelangte das Wort - ins Wogulische und Ungarische getrennt - frühestens aus irgendeiner spät-mitteliranischen Sprachform. Ins Ungarische der Wanderungszeit kam es möglicherweise etwas später als ins Wogulische, wohl durch Vermittlung persischer Handelskontakte. Als Quelle für das wogul. Wort kann kaum eine der in Europa gesprochenen alten iranischen Sprachen gelten, sondern es stammt offenbar aus jenen mitteliranischen Dialekten, die in Westsibirien bis zum Altaigebirge gesprochen wurden und die ausser in den obugrischen Sprachen auch im Samojedischen und in den türkischen Mundarten Sibiriens Spuren hinterlassen haben.

Von jenen 44 Etymologien, die in der oben genannten, soweit bekannt bald im Druck erscheinenden Untersuchung von Éva Korenchy ${ }^{1}$ enthalten sind, betrifft ungefähr die Hälfte solche als iranisch erklärten Wörter, die lediglich auf die obugrischen Sprachen beschränkt sind. Ohne die Arbeit von Éva Korenchy hier detaillierter zu referieren oder zu ergänzen möchte ich in diesem Zusammenhang nur auf ein paar, in ihrem Manuskript fehlende Wortvergleiche aufmerksam machen, die auch schon früher in der Fachliteratur teilweise berührt worden sind.

Das gemeinwogulische Nomen TJ, TČ $\varepsilon t ə \cdot r, \mathrm{KU}, \mathrm{KM}, \mathrm{KO}$, VN, VS ätar, P ectar, LU ctar, LO, So atar 'klar; heiteres Wetter' hat im Ostjakischen etar, etar, èter, ătar 'klar, hell, heiter(Wetter Tag, Himmel); klares Wetter; trockene Witterung' (s. Steinitz, DEWb. Sp. 218-219) eine genaue Entsprechung. Paasonen hat diese Wörter zusammengestellt (Ostj.Wb. 20) und die Bezeichnung »lpK» hinzugefügt, womit er offenbar das im Kolalappischen Wörterbuch von Genetz stehende Substantiv vierhta 'klares Wetter, heller Himmel' meint. Steinitz hat es (a.a.O.) nicht für nötig befunden, diese Etymologie hinsicht-

\footnotetext{
1 Während dieser Aufsatz gesetzt wurde, erschien Korenchys Untersuchung in deutscher Sprache im Druck: Iranische Lehnwörter in den obugrischen Sprachen (Budapest 1972).
} 
lich des Lappischen zu wiederholen; sie ist auch eindeutig falsch. Toivonen hat (FUF XXX 347) das diesbezügliche lappische Wort behandelt, das im gesamten Sprachgebiet angetroffen wird (z.B. U veärrdoo, N vier'to, fier'to, T viè̈'ta). Er hält es für ein wahrscheinlich germanisches (skand.) Lehnwort: an. vedr, aschw. vceper, schw. väder, ahd. wetar, dt. Wetter usw. (< germ. *uedra- 'Wind, Wetter'). - Der etymologische Hinweis in Paasonens Wörterbuch endet mit einer Bemerkung, die Steinitz ebenfalls weggelassen hat: »cf. skr. vidue bei hellem himmel, UHL. 291». Durch einen Druckfehler ist das Sanskritwort ganz unerkennbar geworden: es müsste vìdhré heissen, der Lok. Sg. vom Adjektiv vīdhráh 'rein, klar' $\left(<v i+{ }^{*} i d h-r a ́\right.$; vgl. auch aind. idhriyah 'zum heiteren Himmel gehörig'; s. Mayrhofer, Aind. Etym.Wb. III 237, I 95, 557). Paasonens Bemerkung wurde von Georg Morgenstierne aufgenommen. In seinem Wörterverzeichnis der in Afghanistan gesprochenen iranischen Sprachen yidghā und munji (Indo-Iran. Frontier Lang. II 224 a) stellt er fest, dass möglicherweise jene Wort-

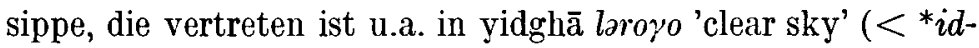
rakā) und yudur ( $\left.{ }^{*} e d h r a-\right)$ id. im dardischen khowar, in die fiu. Sprachen entlehnt sei (ins Ostjakische und Kolalappische, - hier verweist er auch auf eine Information von Collinder). Das iranische Wort beschränkt sich nicht ausschliesslich auf die Pamir-Gegenden, sondern es besitzt u.a. auch im Ossetischen eine Entsprechung: ird 'hell, klar' (< *idra bzw. *vidra; vgl. V.I. Abaev, Историко-әтимол. словарь осетинск. яз. I 547-548 und E. Benveniste, Études sur la langue ossète 96). - Es ist durchaus möglich, dass das obugrische * ětar 3 aus irgendeiner mitteliranischen Sprachform entlehnt ist.

Das wogul. Substantiv mant wird in den Wörterbüchern der heutigen Schriftsprache übersetzt mit 'лопата; Schaufel'. Béla Kálmán hat aus den Dialekten folgende Formen aufgezeichnet: Sygva mānt, mānt, Ob mānt 'kurzer schaufelförmiger Stock zur Reinigung der Skier und Kleider von Schnee, zum Hervorgraben der Rentierflechte unter dem Schnee, zur Reinigung der Schlafstelle oder der Feuerstätte usw.' (briefl. Mitteilung). Ernst Lewy hat dieses wogul. Wort mit aind. mánthās 'Rührstock' verglichen. Mayrhofer erwähnt (Aind. Etym. Wb. II 
578-580) s.v. aind. mánthati 'quirlt, rührt, zermalmt, reibt, schüttelt' u.a. die folgenden Formen: manthá- m. 'Rührtrank', (Akk. Sg.) mánthãm 'Stössel', mánthitar- 'Rührer, Schüttler'. Turner wiederum zählt (Comp.Dict. Nr. 9841) z.B. sanskr. manthá- 'stirring spoon', pahārī manth 'churning stick' auf. Diese indoarische Wortfamilie ist identisch mit iranisch *man $\bar{p}$ -

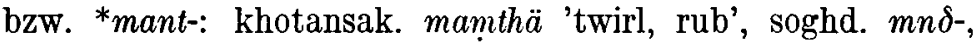
osset. iron azmantyn, digor cezmantun 'zusammenmischen', parāčĩ menth- 'to rub', yidghā lamōn- id.; sarīkolī mā 'Stock, Stöckchen, Stab', šughnī mâ $\vartheta$, yidghā mōxë id. (s. zuletzt H. W. Bailey, Khotanese Texts VI 274). - Nichts hindert uns, das wogulische Wort, dessen frühere Lautgestalt *mant(3) oder (?) *mänt(3) hat sein können, für arisch, hauptsächlich iranischen Ursprungs, zu halten. ${ }^{1}$

In den obugrischen Sprachen gibt es noch einige andere Namen von Werkzeugen, Waffen und Geräten, die als Übernahmen von iranischer Seite ausgelegt werden können. Hierher gehören - in der Literatur schon früher behandelt - u.a. wog.

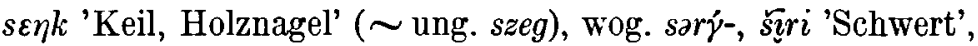
wog. śóa 'Keule, Hammer' ( ostj. śá ). Toivonen hat (FUF XX 58) auch das ostj. Substantiv pili, pila, piliw 'Spaten, Schaufel' auf iranischen Ursprung zurückgeführt und es (wie schon Paasonen, Ostj. Wb. 182) u.a. mit dem neupers. Wort $b \bar{e} l, b \bar{\imath} l$ id. verglichen. Glaubhafter ist jedoch, dass es sich nicht um eine direkte iranische, sondern durch Vermittlung der Türksprachen Sibiriens erhaltene Entlehnung handelt, vgl. z.B. osm. bäl 'Hacke, Kelt', kirg., kumyk. bel, trkm. pil 'Spaten, Schaufel' (genauer: Räsänen, Etym.Wb. der Türksprachen 69). - Durch Vermittlung der Türksprachen gelangte eine Reihe neuiranischer Lehnwörter auch in die samojedischen Sprachen, vor allem in die südlichen (s. MSFOu 103 44). Ausserdem gibt es im Samojedischen einige verbreitetere und of-

${ }^{1}$ Aus irgendeiner anderen mitteliranischen Quelle ist offenbar mordM mandă, E mando 'Stock, Stab' entlehnt worden, worauf Erkki Itkonen mich freundlicherweise aufmerksam gemacht hat. Aus dem baltischen Zweig dieser idg. Wortsippe, der u.a. vertreten ist durch lit. meñte 'Quirl, Mauerkelle, Schaufel, Spaten', ist z.B. fi. mäntä 'Kolben; (Butter-) Stössel; Quirl' entlehnt worden (s. SKES 359). 
fensichtlich ältere Lehnwörter der mitteliranischen Epoche, die chronologisch und hinsichtlich ihrer Quelle offenbar mit der hier behandelten mitteliranischen Schicht der obugrischen Sprachen zusammengehören. Solche Fälle sind u.a. samJur. pul, pur 'Brücke', Jen. fúl' $u$, fúru', T fûli, slk. pâl, pyl, kam. pülü $\sim$ pehl. puhl, npers. pul usw. id. (vgl. Verba docent 50 Fussn. 3); sam'T tagai 'Messer', kam. tagai, dagai 'id.; Speer, Spiess', koib. tagai 'Messer' (> tat. Sibir. ta $>$ ai 'Waffe'), slk. teaga, teäga, täka 'Schwert; Speer, Spiess' $\sim$ awest. taē $\gamma a-$

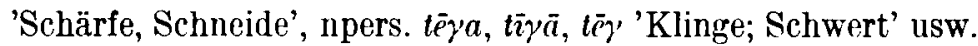
(s. MSFOn 103 302/3).

Einen langen und teilweise noch unerforschten Weg bis ins Nordwogulische hinein hat jene Metallbezeichnung zurückgelegt, die die Wörterbücher der Gegenwartssprache in der Form анах 'олово', 'Zinn' notieren. In Kannistos Wörtersammlungen begegnet es (nach der freundl. Mitteilung von Matti Liimola) lediglich in den zwei nördlichsten Dialekten: LO ana $\chi$ : $\dot{a} .-\bar{a} t-$

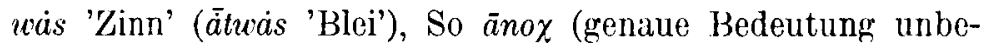
kannt). Bernát Munkácsi - reich an Ideen und rasch im Schreiben - hat in seinem Artikel "Spuren eines assyrischen einflusses auf finnisch-magyarische sprachen» (KSz XII 261/71) neben zahlreichen Fehlschlägen offenbar doch das Richtige getroffen, wenn er meint, das in Frage stehende Wort im Wogulischen stamme aus Vorderasien. - Der älteste bekannte Beleg findet sich in den kappadokischen Urkunden um 2000 v.Chr.: akkadisch anāku 'Zinn'. Hieraus sind u.a. hebr. 'anāk, syr. 'ankhā entlehnt worden sowie weiter arab. 'ānuk, äthiop. $n \bar{a} ' i k$, armen. anag, altind. nāgam 'Blei, Zinn' (vgl. Armas Salonen, StO XVII:2 6; Mayrhofer, Aind. Etym. Wb. II 150). Aus dem Altindischen gelangte das Wort in einige andere indoarische Sprachen: dardisch (shina) nan m. 'Blei', kaschmirisch $n \bar{a} g$ m. id. (Turner, Comp. Dict. Nr. 7040). Vermutlich haben armenische oder jüdische Kaufleute diesen semitischen $\mathrm{Me}-$ tallnamen nach Westsibirien gebracht. Wegen der spärlichen Quellen bleibt ungeklärt, ob es die Iraner, dic Tataren oder erst die Russen waren, die es den Wogulen vermittelten. 